\title{
Applications of Fuzzy Logic, Artificial Neural Network and Neuro-Fuzzy in Industrial Engineering
}

\author{
Raheleh Jafari ${ }^{1[0000-0001-7298-2363]}$, Miguel A. Contreras ${ }^{2}$, Wen Yu ${ }^{2}$ \\ Alexander Gegov 1[0000-0002-6166-296X] \\ ${ }^{1}$ School of design, university of Leeds, LS2 9TJ, Leeds, UK \\ r.jafari@leeds.ac.uk \\ ${ }^{2}$ School of Engineering and science,Tecnologico de Monterrey,Prol.Juan de la Bar- \\ rera1241,Saltillo25270, Mexico \\ macontretec.mx \\ ${ }^{3}$ Departamento de Control Automatico CINVESTAV-IPN (National Polytechnic Institute) \\ Mexico City 07360, Mexico \\ yuw@ctrl. cinvestav.mx \\ ${ }^{4}$ School of Computing, University of Portsmouth, Buckingham Building, Portsmouth PO1 \\ 3HE, UK \\ alexander.gegov@port.ac.uk
}

\begin{abstract}
Artificial intelligence methods namely artificial neural network, fuzzy logic, and neuro-fuzzy have been effectively utilized in different applications like business, marketing, control engendering, health care, and social services. To demonstrate the usage of fuzzy set theory, artificial neural network, as well as neuro-fuzzy in industrial engineering and also for providing a basis for future investigation, a literature review of the artificial neural network, fuzzy logic, and neuro-fuzzy in industrial engineering is conducted in this paper.
\end{abstract}

Keywords: Fuzzy Logic, Artificial Neural Network, Neuro-Fuzzy, Industrial Engineering.

\section{Introduction}

Industrial engineering is one of the fields that artificial neural network, fuzzy logic, and neuro-fuzzy have found an extensive implementation area. Artificial neural networks can be considered as the most effective technique over the last few decades that are extensively utilized in a wide variety of implementations in different fields [1-6]. Artificial neural networks have been considered as efficient and versatile tools. They have learning ability as well as model-free characteristics.

Fuzzy systems are appropriate for estimated reasoning, mainly for the system with a mathematical design which is hard to obtain [7-11]. Fuzzy set theory can be considered as a major problem designing as well as a solution approach. The main contribution of 
fuzzy logic is its ability to present vague data. Fuzzy logic has been implemented to design systems, which are difficult to define accurately. In recent years, successful implementations of fuzzy logic in industrial engineering have been reported. Industrial engineers encounter with numerous problems having incomplete as well as uncertain information [12-15]. Fuzzy logic theory can be considered as a capable tool for solving these kinds of problems. Fuzzy logic presents an effective tool to facilitate investigation in industrial engineering when the dynamics of the decision environment restrict the accurate evaluation of model parameters.

Neuro-fuzzy has been used in an extensive range of domains [16-18]. It is the combination of artificial neural networks and fuzzy logic. The cause of utilizing artificial neural networks with fuzzy logic is that artificial neural networks never make a presumption on the probability distribution functions of data [19]. Generally, the neurofuzzy model explains solutions better than artificial neural networks [20].

This paper represents details of the application of artificial neural network, fuzzy logic, and neuro-fuzzy in industrial engineering. In this paper, the most recent researches in the field of artificial neural network, fuzzy logic, and neuro- fuzzy are covered. Since some industries have successfully used these techniques, detailed discussions are supplied to stimulate future investigations. This article remaining sections are organized into four Sections. In Section 2 the applications of fuzzy logic in industrial engineering are given. In Section 3 the applications of artificial neural network in industrial engineering are given. Section 4 presents the applications of neuro-fuzzy in industrial engineering. Conclusions are included in Section 5.

\section{Applications of fuzzy logic in industrial engineering}

Fuzzy logic systems have been effectively applied in different industrial fields like automobile speed control [21], robot arm control [22], water quality control [23], and automatic train operation systems [24]. Fuzzy logic can be utilized for improving the efficiency of the system. Even though fuzzy logic is considered as an approach for presenting inaccurate and vague information, in [25] the Universal Approximation Theorem states that the fuzzy logic system can uniformly approximate any nonlinear function to any degree of preciseness.

The fuzzy inference system utilizes fuzzy set theory for mapping inputs to outputs. The common fuzzy inference system is Mamdani and Sugeno type. Fuzzy inference system consists of a fuzzification interface, a rule database, a decision making unit as well as defuzzification interface, see Fig. 1. 


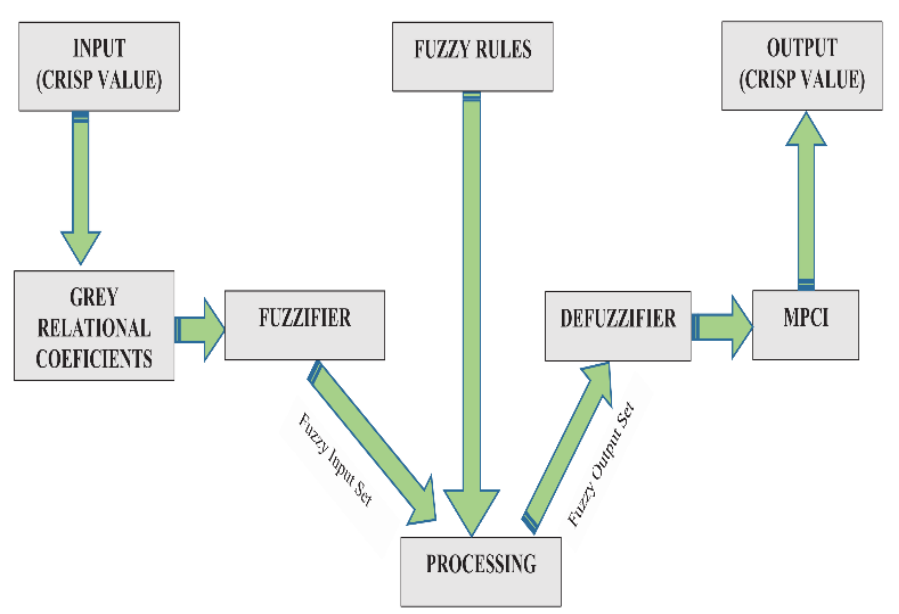

Fig. 1. The flowchart of a fuzzy inference system.

Fuzzy logic systems are extremely effective in highly complicated and nonlinear processes, and also in the lack of any simple mathematical model. In [26], fuzzy set theory is utilized for generating a novel pulse discriminator in electric dis- charge machining procedure. In electric discharge machining procedure, cutting efficiency indexes like material elimination rate as well as surface harshness are in direct relation with the electric discharge machining discharge pulses.

In [27], the fuzzy logic is utilized for control of the fluid catalytic cracking unit. In that paper, the fuzzy logic control as a control method is efficiently applied for improved procedure control of fluid catalytic cracking in the refinery process industry.

\section{Applications of artificial neural network in industrial engineering}

In contrast with the fuzzy system, the neural network is desirable at predicting. This potential originates from the learning ability as well as model-free characteristics. The design of the artificial neural network is based on a group of interconnected artificial neurons along with linear or nonlinear transfer functions. Neurons are placed in various layers such as input layer, hidden layer, as well as output layer. The artificial neural network learns the relevance between input and output of the system employing an iterative procedure named training. For every input into the neuron, there is a weight. Weight is a tune able number that is defined while training the network. A simple model of the artificial neural network is shown in Fig. 2. 


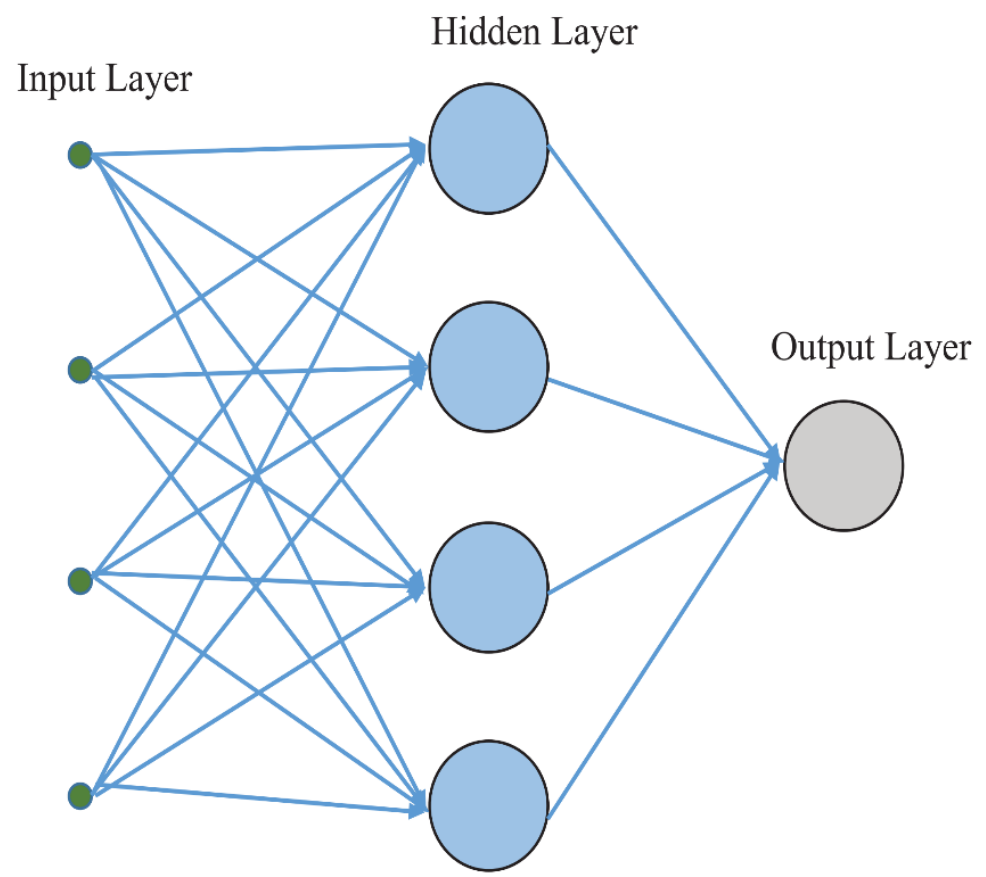

Fig. 2. A simple model of the artificial neural network.

One of the applications of the artificial neural network is in condition monitoring. Artificial neural network provides a reliable procedure to condition monitoring. Condition monitoring can be considered as an effective device in maintenance planning, also it can be utilized for avoiding the unpredicted failures. In [28], artificial neural network technique is used for experimentally recognizing gears as well as bearings faults related to a typical gearbox system.

The artificial neural network has been used for evaluating vibrations as well as recognizing fault existence. Fault detection is considered as a crucial issue for gas turbine owners to move from preventative repair to predictive repair and hence to decrease the repair expenses. In [29], artificial neural network technique is used for fault detection of an industrial gas turbine.

\section{$4 \quad$ Applications of neuro-fuzzy in industrial engineering}

Artificial neural network and fuzzy logic are both considered as model-free numerical techniques. Each technique uses an uncomplicated algorithmic procedure instead of a complex mathematical analysis, and also the parameters are tune- able [30]. These resemblances make it possible to combine the two techniques.

Neuro-fuzzy system is a multi-layer feedforward adaptive network which figures out the fundamental elements as well as functions of a standard fuzzy logic system. As 
fuzzy logic systems are proved to be universal approximators, and since neuro-fuzzy systems are isomorphic to standard fuzzy logic systems concerning their functions hence neuro-fuzzy systems are likewise universal approximators [31]. For a special method in neuro-fuzzy, we can refer to the adaptive neuro-fuzzy inference system which is considered as one of the initial integrated hybrid neuro-fuzzy models [32].

One of the applications of the neuro-fuzzy system is in polymerization systems. Over the last few decades solubility of gases in polymers has been of interest to chemical engineers. In [33], a hybrid grid partitioning adaptive neuro-fuzzy inference system is utilized to predict carbon dioxide solubility in polymers.

The major aim of current manufacturing industries is to manufacture low cost, superb quality products in less time [34]. The choice of optimal cutting parameters is crucial in each machining procedure for increasing the quality of machined productions as well as to decrease the machining expenses. In [35], an adaptive neuro-fuzzy inference system is introduced to model and predict surface roughness in ball end milling of a die material. The algorithm developed in that paper presets the cutting parameters for a favorable level of surface roughness.

\section{Conclusion}

In this paper, the recent advances of the artificial neural network, fuzzy logic, and neuro-fuzzy applications in industrial engineering are provided. The artificial neural network, fuzzy logic, and neuro-fuzzy are the three major computational intelligence methods. Utilization of these models can be taken into account as a cheap, highly effective, and more reliable alternative devices. Therefore, these three methods can provide more ability to problem resolving than other techniques.

\section{References}

1. Razvarz, S., Jafari, R., Yu, W., Khalili, A.: PSO and NN Modeling for Photocatalytic Removal of Pollution in Wastewater, 14th International Conference on Electrical Engineering, Computing Science and Automatic Control (CCE) Electrical Engineering, $1-6,(2017)$.

2. Jafari, R., Yu, W.: Artificial neural network approach for solving strongly degenerate parabolic and burgers-fisher equations, 12th International Conference on Electrical Engineering, Computing Science and Automatic Control, doi:10.1109/ICEEE.2015.7357914, (2015).

3. Jafari, R., Razvarz, S., Gegov, A.: A New Computational Method for Solving Fully Fuzzy Nonlinear Systems, In: Computational Collective Intelligence. ICCCI 2018. Lecture Notes in Computer Science, Springer, Cham, 11055, 503-512 (2018).

4. Razvarz, S., Jafari, R.: ICA and ANN Modeling for Photocatalytic Removal of Pollution in Wastewater, Mathematical and Computational Applications, 22, 38-48, (2017). 
5. Razvarz, S., Jafari, R., Gegov, A., Yu, W., Paul, S.: Neural network approach to solving fully fuzzy nonlinear systems, Fuzzy modeling and control Methods Application and Research, Nova science publisher, Inc, NewYork. ISBN: 978-1-53613- 4155, 45-68 (2018).

6. Razvarz, S., Jafari, R.: Intelligent Techniques for Photocatalytic Removal of Pollution in Wastewater, Journal of Electrical Engineering, 5, 321-328 (2017). doi: 10.17265/2328-2223/2017.06.004.

7. Jafari, R., Razvarz, S.: Solution of Fuzzy Differential Equations using Fuzzy Sumudu Transforms, IEEE International Conference on Innovations in Intelligent Systems and Applications, 84-89 (2017).

8. Jafari, R., Razvarz, S., Gegov, A., Paul, S.: Fuzzy modeling for uncertain nonlinear systems using fuzzy equations and Z-numbers, Advances in Computational Intelligence Systems: Contributions Presented at the 18th UK Workshop on Computational Intelligence, September 5-7, 2018, Nottingham, UK . Advances in Intelligent Systems and Computing, Springer, 840, 66-107 (2018).

9. Jafari, R., Razvarz, S.: Solution of fuzzy differential equations using fuzzy sumudu transforms, Mathematical and Computational Applications, 1-15 (2018).

10. Jafari R., Razvarz S., Gegov A.: Solving Differential Equations with Z-Numbers by Utilizing Fuzzy Sumudu Transform, Intelligent Systems and Applications. IntelliSys 2018. Advances in Intelligent Systems and Computing, Springer, Cham. 869, 1125 1138 (2019).

11. Yu, W., Jafari, R.: Fuzzy Modeling and Control with Fuzzy Equations and ZNumber, IEEE Press Series on Systems Science and Engineering, Wiley-IEEE Press, ISBN-13: 978-1119491552, (2019).

12. Razvarz, S., Jafari, R.: Experimental study of Al2O3 nanofluids on the thermal efficiency of curved heat pipe at different tilt angle, Journal of Nanomaterials, 1-7, (2018).

13. Razvarz, S., Vargas-Jarillo, C., Jafari, R.: Pipeline Monitoring Architecture Based on Observability and Controllability Analysis, IEEE International Conference on Mechatronics (ICM), Ilmenau, Germany, 1, 420-423, (2019) doi:10.1109/ICMECH.2019.872287,.

14. Razvarz, S., Vargas-jarillo, C., Jafari, R., Gegov, A.: Flow Control of Fluid in Pipelines Using PID Controller, IEEE Access, 7, 25673-25680, (2019) doi:10.1109/ACCESS.2019.2897992.

15. Jafari, R., Yu, W., Razvarz, S., Gegov, A.: Numerical methods for solving fuzzy equations: A Survey. In: Fuzzy Sets and Systems, ISSN 0165-0114, (2019) https://doi.org/10.1016/j.fss.2019.11.003. .

16. Jafari, R., Yu, W., Li, X.: Solving Fuzzy Differential Equation with Bernstein Neural Networks, IEEE International Conference on Systems, Man, and Cybernetics, Budapest, Hungary, 1245-1250 (2016).

17. Jafari, R. Yu, W.: Uncertain nonlinear system control with fuzzy differential equations and Z-numbers, 18th IEEE International Conference on Industrial Technology, Canada, 890-895 (2017). doi:10.1109/ICIT.2017.7915477. 
18. Jafarian, A., Measoomy nia, S., Jafari, R.: Solving Fuzzy Equations Using Neural Nets with a New Learning Algorithm, Journal of Advances in Computer Research, 3, 33-45 (2012).

19. Castellano, G., Fanelli, A.M.: Variable selection using neural-network models, Neuro computing, 31, 1-13 (2000).

20. Babuska, R., Verbruggen, H.: Neuro-fuzzy methods for nonlinear system identification, Annual Reviews in Control, 27, 73-85 (2003).

21. Murakami, S., Maeda, M.: Automobile speed control system using a fuzzy logic controller, M. Sugeno (Ed.), Industrial Applications of Fuzzy Control, North-Holland, Amsterdam, (1985).

22. Scharf, E.M., Mandix, N.J.: The application of a fuzzy controller to the control of a multi-degree-of-freedom robot arm, M. Sugeno (Ed.), Industrial Applications of Fuzzy Control, North-Holland, Amsterdam, (1985).

23. Yagishita, O., Itoh, O., Sugeno, M.: Application of fuzzy reasoning to the water purification process, M. Sugeno (Ed.), Industrial Applications of Fuzzy Control, NorthHolland, Amsterdam, (1985).

24. Yasunobu, S., Miyamoto, S.: Automatic train operation system predictive fuzzy control, M. Sugeno (Ed.), Industrial Applications of Fuzzy Control, North- Holland, Amsterdam, (1985).

25. Wang, L.X.: Adaptive Fuzzy Systems and Control, Design and Stability Analysis, Prentice Hall, NJ, (1994).

26. Tarng, Y.S., Tseng, C.M., Chung, L.K.: A Fuzzy Pulse Discriminating System For Electrical Discharge Machining, International Journal of Machine Tools and Manufacture, 37, 511-522, (1997).

27. Taskin, H., Cemalettin, K., Uygun, O., Arslankaya, S.: Fuzzy logic control of a fluid catalytic cracking unit (FCCU) to improve dynamic performance, Computers and Chemical Engineering, 30, 850-863, (2006).

28. Rafiee, J., Arvani, F., Harifi, A., Sadeghi, M.H.: Intelligent condition monitoring of a gearbox using artificial neural network, Mechanical Systems and Signal Processing, (2006).

29. Arriagada, J., Genrup, M., Assadi, M., Loberg, A.: Fault Diagnosis System for an Industrial Gas Turbine by Means of Neural Networks, International Gas Turbine Congress, Tokyo, Japan, (2003).

30. Bersini, H., Nordvik, J., Bonarini, A.: A simple direct adaptive fuzzy controller derived from its neural equivalent, Proc. 1993 IEEE Int. Conf. on Fuzzy System, 1, 345-350, (1993).

31. Wang, L.X., Mendel, J.M.: Fuzzy basis functions, universal approximation, and orthogonal least squares learning, IEEE Transactions on Neural Networks, 3, 807- 814 (1992).

32. Jang, J.: ANFIS: Adaptive network-based fuzzy inference systems, IEEE Transactions on Systems Man and Cybernetics, 23, 665-685 (1993).

33. Khajeh, A., Modarress, H., Rezaee, B.: Application of adaptive neuro-fuzzy inference system for solubility prediction of carbon dioxide in polymers, Expert Systems with Applications, 36, 5728-5732 (2009). 
34. Yazdi, M.R.S., Khorram, A.: Modeling and Optimization of Milling Process by using RSM and ANN Methods, International Journal of Engineering and Technology (IJET), IACSIT, 2, 474-480 (2010).

35. Hossain, Md. Sh. J., Ahmad, N.: A Neuro-fuzzy Approach to Select Cutting Parameters for Commercial Die Manufacturing, Procedia Engineering, 90, 753-759 (2014). 